\title{
Atenção nutricional e ganho de peso gestacional em adolescentes: uma abordagem quantiqualitativa
}

\author{
Nutritional care and weight gain in pregnant adolescents: \\ a quantitative and qualitative approach
}

\author{
Marta Maria Antonieta de Souza Santos ${ }^{1}$ \\ Denise Cavalcante de Barros ${ }^{2}$ \\ Mirian Ribeiro Baião ${ }^{1}$ \\ Claudia Saunders ${ }^{1}$
}

${ }^{1}$ Departamento de Nutrição Social Aplicada, Instituto de Nutrição Josué de Castro, Universidade Federal do Rio de Janeiro. Av. Brigadeiro Trompowisky CCS/Bl. J/2\%/sala 07, Ilha do Fundão. 00000-000 Rio de Janeiro RJ.

antonietamss@globo.com

${ }^{2}$ Escola Nacional de Saúde

Pública/Fundação Oswaldo

Cruz.

\begin{abstract}
This study sought to evaluate the impact of nutritional assistance (intervention) on total gestational weight gain in adolescents and to understand the perceptions of adolescents regarding the intervention. It is a quantitative and qualitative survey using triangulation methods conducted in a public maternity hospital in Rio de Janeiro with 204 adolescents, divided into preintervention and intervention groups. The hypothesis of homogeneity of proportions was tested by bivariate and multivariate analysis using Poisson regression with robust variance. The qualitative approach was conducted using semi-structured interviews with 12 adolescents, whose data were submitted to content analysis. The results indicate that the intervention did not have a positive effect on the adequacy of total gestational weight gain. The adolescents perceived the dietary plan as a set of rules incompatible with their conditions as pregnant women. The category "almost every consultation was the same" emerged as a synthesis of the perceptions on the intervention. It was concluded that there is a need to reassess the technical and scientific marker tools of nutritional assistance and turn it into care focused around making shared decisions.
\end{abstract}

Key words Weight gain, Pregnancy in adolescence, Nutritional status
Resumo Neste estudo procurou-se avaliar o impacto da assistência nutricional (intervenção) sobre o ganho de peso gestacional total de adolescentes e compreender a percepção destas sobre a intervenção. Trata-se de uma investigação quantiqualitativa com triangulação de métodos, realizada em uma maternidade pública do Rio de Janeiro com 204 adolescentes, subdivididas nos grupos pré-intervenção e intervenção. A hipótese de homogeneidade de proporções foi testada por análises bi e multivariada, por meio de regressão de Poisson com variância robusta. Na abordagem qualitativa realizou-se entrevista semiestruturada com 12 adolescentes, cujos dados foram submetidos à análise de conteúdo. Os resultados apontam que a intervenção não exerceu efeito positivo na adequação do ganho de peso gestacional total. As adolescentes perceberam o plano alimentar como um conjunto de regras incompatíveis com suas condições de grávidas. A categoria "quase toda consulta era a mesma coisa" surgiu como sintese da percepção sobre a intervenção. Concluiu-se que há necessidade de reavaliar o instrumental tecnocientífico balizador da assistência nutricional e transformá-la em cuidado centrado na tomada de decisões compartilhadas.

Palavras-chave Ganho de peso, Gravidez na adolescência, Estado nutricional 


\section{Introdução}

A gravidez na adolescência é um fenômeno complexo, tanto do ponto de vista biológico quanto psicossociocultural. Os estudiosos desse tema têm considerado o seu significado e suas implicações subjetivas. Afinal, essa questão, repleta de singularidades, continua sendo desafiadora para as famílias, os profissionais de saúde, os educadores, as sociedades, e para os próprios adolescentes. Nesse sentido, o estudo da gravidez na adolescência tem interessado a grupos cada vez maiores de pesquisadores das diversas áreas do conhecimento, incluindo a Nutrição.

Diversos estudos ${ }^{1-3}$ apontam a estreita relação entre estado nutricional da adolescente, antes e durante a gestação, com o ganho de peso gestacional total. Também é consenso entre pesquisadores $^{4-8}$, e entre profissionais de saúde, que o baixo peso materno pré-gestacional e o insuficiente ganho de peso durante a gestação podem exercer influência negativa sobre o estado clínico da gestante, agravando problemas obstétricos, bem como aumentar o risco de retardo intrauterino e morbimortalidade perinatal. De forma semelhante, o excessivo ganho de peso gestacional total tem sido associado à maior ocorrência de parto cirúrgico e ao aumento das taxas de complicações no parto, macrossomia fetal, desenvolvimento de síndromes hipertensivas e diabetes mellitus gestacional e malformação congênita fetal ${ }^{4,6,8}$.

A adequação do ganho de peso gestacional total (GPGT) é uma preocupação dos comitês de saúde, que têm sugerido medidas de promoção da sua adequação ${ }^{9-11}$. No estudo de Olson e Strawderman ${ }^{12} 25 \%$ das gestantes que tiveram GPGT acima das recomendações do Insitute of Medicine $(\mathrm{IOM})^{13}$ retiveram, no mínimo, $4,5 \mathrm{~kg}$ no pósparto. O estudo de Polley et al. ${ }^{14}$ concluiu que a inadequação do GPGT pode ser minimizada com a intervenção nutricional, uma vez que gestantes que receberam orientações quanto à prática de exercícios e alimentação saudável na gestação tiveram menor prevalência de ganho de peso acima do recomendado $(33 \%)$ do que as que não receberam a intervenção (58\%).

Contudo, apesar das evidências científicas apontarem a necessidade de estratégias para promoção da adequação do ganho de peso gestacional total, de maneira a impactar positivamente no resultado obstétrico, Rouse ${ }^{15}$ destaca que a intervenção nutricional no pré-natal (PN) ainda não foi alvo de avaliação formal nos países em desenvolvimento. Ademais, a assistência nutrici- onal, de modo geral entendida como fenômeno que estabelece condutas, não pode se eximir da interpretação dos aspectos subjetivos, individuais e coletivos, relacionados ao comer e à comida. Neste sentido, para além dos fatores biológicos, o cuidado nutricional das gestantes adolescentes deve considerar a pluralidade de formas, de possibilidades e de significações de como a orientação nutricional é percebida e praticada por essas jovens.

Este manuscrito descritivo-interpretativo com abordagem quantiqualitativa objetiva avaliar $\mathrm{o}$ impacto da assistência nutricional sobre o GPGT de adolescentes, antes e após a implantação de um protocolo de assistência nutricional PN (intervenção), e compreender os sentidos e significados dessa assistência para essas adolescentes.

\section{Procedimentos metodológicos}

Trata-se de um estudo de abordagem quantiqualitativa, fundamentada nos princípios da triangulação de métodos, visando não apenas caracterizar os resultados em nível descritivo-analítico, mas, acrescentar-lhes significados em busca da compreensão dos fenômenos segundo a perspectiva das adolescentes ${ }^{16,17}$. Cabe aqui destacar que dentre os diferentes referenciais teóricos e as perspectivas sob as quais a adolescência vem sendo amplamente estudada, no presente estudo optou-se por compreender e interpretar os fenômenos apreendidos sob a orientação do referencial das Ciências Sociais.

A investigação foi realizada no período de novembro/2007 a fevereiro/2010 em uma maternidade pública do município do Rio de Janeiro, que dispõe de ambulatórios de PN de baixo e alto risco e atende gratuitamente gestantes adultas e adolescentes, residentes no município. A maternidade recebe gestantes em trabalho de parto que tenham feito ou não o $\mathrm{PN}$ na unidade e realiza uma média mensal de 220 a 230 partos.

$\mathrm{Na}$ primeira fase do estudo foi realizada a descrição do perfil de 160 puérperas adolescentes, admitidas em trabalho de parto na maternidade estudada no período anterior à intervenção proposta (Grupo I - pré-intervenção GI), para as quais havia o registro de informações em prontuário sobre história obstétrica e clínico-nutricional, variáveis sociodemográficas e da assistência PN.

Na segunda fase foi aplicada a intervenção a todas as gestantes adolescentes presentes na maternidade no período de captação (novembro/ 
2007 a julho/2009), e que atenderam aos critérios de elegibilidade e aceitaram participar do estudo. Todas as participantes maiores de 18 anos de idade, e os responsáveis pelas menores, assinaram termo de consentimento obtido de forma livre e espontânea. Foi composta uma coorte de 204 adolescentes, com idade inferior a 20 anos, com gestação de feto único e não portadoras de enfermidades com início prévio à gestação. O grupo foi acompanhado desde o início do $\mathrm{PN}$ até o puerpério imediato, constituindo-se no grupo intervenção (GII). Todas as adolescentes incluídas na coorte tiveram seus partos até fevereiro/2010.

A principal característica da intervenção era o cuidado nutricional individualizado, com aconselhamento dietético personalizado, oferecido em um mínimo de 4 consultas com o nutricionista ao longo da gestação, intercaladas por três atendimentos em grupo. Em cada consulta individual eram realizadas as avaliações: nutricional (antropométrica, dietética, clínica, funcional, bioquímica), dos exames complementares, das condições sociodemográficas, da história obstétrica e da alimentação habitual da gestante. A ocorrência de interdições e intolerâncias alimentares foi investigada por meio da avaliação detalhada do padrão e composição das refeições.

Para a efetivação do cuidado nutricional foram considerados os princípios do aconselhamento dietético, enquanto uma proposta educativa visando à sustentação da reorientação da praxis do nutricionista para transformá-la em um encontro dialogado e horizontalizado com a adolescente, de forma a contemplar as dimensões subjetivas e educativas do agir em nutrição ${ }^{18,19}$.

Em março de 2008, quando começaram a ocorrer os primeiros partos, deu-se início à terceira fase da investigação. Com caráter exploratório, esta se desenvolveu simultaneamente à segunda fase e se caracterizou pela aplicação da técnica de entrevista semiestruturada às adolescentes. Foram realizadas 12 entrevistas, obedecendo ao critério de saturação dos sentidos.

A quarta e última fase da pesquisa foi dedicada à análise crítica e à busca da ampliação da qualidade dos dados e da validade das interpretações produzidas. Os dados quantitativos foram digitados no pacote estatístico Statistical Package for the Social Sciences (SPSS for windows versão 17.0).

Adotou-se a recomendação do Ministério da Saúde ${ }^{20}$ para avaliação da adequação do ganho de peso gestacional total, considerando-se as faixas de ganho de peso segundo o estado nutricional pré-gestacional (Índice de Massa Corporal -
IMC $\mathrm{kg} / \mathrm{m}^{2}$ ), sendo classificada em duas categorias: ganho adequado e inadequado (insuficiente ou excessivo). Considerou-se adequado quando o ganho de peso se manteve dentro das faixas propostas segundo as categorias de IMC prégestacional $\left[<19,8 \mathrm{~kg} / \mathrm{m}^{2}\right.$ (baixo peso) - 12,5 a $18 \mathrm{~kg} ; 19,8$ a $26,0 \mathrm{~kg} / \mathrm{m}^{2}$ (eutrofia) - 11,5 a 16,0 $\mathrm{kg} ; 26,0$ a 29,0 kg (sobrepeso) - 7 a 11,5 kg; > 29,0 $\mathrm{kg} / \mathrm{m}^{2}$ (obesidade) - 7 a 9,1 kg]. Empregou-se os pontos de corte recomendados pela Organização Mundial da Saúde ${ }^{21}$, segundo a idade em anos e meses para meninas. Na interpretação do padrão de IMC adotou-se a classificação proposta pelo Ministério da Saúde ${ }^{22}$ adaptada por Saunders et al. ${ }^{4}$.

As variáveis independentes incluídas nas análises foram: (1) sociodemográficas: idade materna, cor da pele (identificada por autoclassificação em branca e parda ou preta), situação marital (solteira; casada ou vive com o companheiro), nível de instrução (ensino fundamental incompleto; ensino fundamental completo; ensino médio incompleto; ensino médio completo ou mais) e condições de saneamento da moradia (adequada - quando apresentava água tratada e encanada, rede de esgoto e coleta de lixo regular; inadequada - quando um desses serviços estava ausente); (2) obstétricas: idade gestacional na primeira consulta do PN (calculada pela Data da Última Menstruação), número de gestações, de partos e de abortos, intervalo interpartal e intergestacional, número de consultas de $\mathrm{PN}$ ( 1 a 5; 6 ou mais) e número de consultas de assistência nutricional PN (1 a 3; 4 ou mais); (3) antropométricas: estatura ( $<1,45 \mathrm{~m} ; 1,45$ ou maior), estado nutricional pré-gestacional segundo IMC (baixo peso; adequado; sobrepeso; obesidade).

Para a estimativa do GPGT e construção da variável dependente do estudo - adequação do GPGT - foram selecionadas 160 adolescentes do GI e 144 do GII, para as quais se dispunha dos dados de peso pré-gestacional e de peso pré-parto ou do registrado na última consulta PN.

Os dados do desfecho de interesse foram analisados e a comparação entre os grupos de estudo (GI e GII) foi realizada como em um estudo transversal. As etapas da análise incluíram respectivamente: descrição da amostra quanto às variáveis investigadas, empregando-se os testes t-Sudent para médias e qui-quadrado para frequências adotando-se o nível de significância de 5\%; avaliação da similaridade entre os grupos GI e GII e; análise bivariada para identificação das variáveis associadas ao desfecho (adequação do ganho de peso gestacional total), com base nas 
razões de prevalência (RP) e intervalos de confiança de 95\%, segundo os grupos de estudo. A seguir, realizou-se a análise multivariada para estimativa da RP ajustada, por meio de regressão de Poisson com variância robusta, utilizando os grupos de estudo como variável independente, além de serem testadas outras variáveis identificadas previamente na análise bivariada, como características de diferenças entre os grupos ou associadas ao desfecho.

A análise qualitativa, sob a perspectiva do paradigma interpretativista ${ }^{23}$, buscou a instauração do diálogo e o resgate dos vínculos entre as informações e suas significações. O processo de análise dos depoimentos foi orientado por uma adaptação essencialmente temática da Análise de Conteúdo proposta por Bardin ${ }^{24}$.

Na fase de "pré-análise", a leitura e a releitura do material empírico permitiram o levantamento de questões e a formulação de hipóteses. $\mathrm{Na}$ etapa seguinte ("exploração do material”), se processou a redução e a organização dos relatos, segundo os temas centrais que emergiram do material empírico, para identificação das categorias êmicas. A categoria "quase toda consulta era a mesma coisa” surgiu como a síntese das percepções das entrevistadas sobre a consulta de nutrição no PN.

$\mathrm{Na}$ etapa interpretativa, o diálogo entre o material empírico e os conhecimentos teóricos possibilitou a compreensão dos significados subjetivos dos depoimentos das adolescentes. A seguir, foi realizado um exercício de triangulação entre informações quantitativas e análise de percepções dessas adolescentes.

Todas as adolescentes que aceitaram participar da entrevista assinaram o Termo de Consentimento Livre e Esclarecido (TCLE) específico para essa etapa. No caso das menores de 18 anos, o TCLE era assinado pelo responsável.

A investigação foi aprovada por um Comitê de Ética em Pesquisa da Universidade Federal do Rio de Janeiro (UFRJ) e consentido pela instituição cenário da pesquisa.

\section{Resultados e discussão}

\section{Caracterização dos sujeitos do estudo}

A maior parte das adolescentes, em ambos os grupos de estudo (Tabela 1), vivia em domicílios com adequadas condições de saneamento e, com frequências similares, consumiram álcool durante a gestação.
Verificou-se que no GII havia maior concentração de adolescentes casadas ou vivendo com companheiro ( $\mathrm{p}<0,001)$, com níveis de instrução mais elevados ( $p<0,001)$, que se autodeclararam pardas ou pretas $(\mathrm{p}<0,001)$ e que faziam uso de cigarro na gestação $(\mathrm{p}=0,016)$. A distribuição das adolescentes segundo o estado nutricional pré-gestacional e a adequação do GPGT não apresentou diferença significativa entre os grupos $(\mathrm{p}>0,05)$.

\section{Avaliação do efeito da intervenção sobre o ganho de peso durante a gestação - a particularidade da gestação na adolescência}

Na Tabela 2 verifica-se similaridade entre GI e GII quando comparados segundo as médias das variáveis idade materna no parto $(\mathrm{p}=0,104)$, número de gestações $(\mathrm{p}=0,122)$, intervalo entre a última gestação e a atual ( $\mathrm{p}=0,613)$ e IMC prégestacional $(\mathrm{p}=0,091)$. Todavia, identificou-se que as adolescentes do GI iniciaram o PN mais precocemente que as do GII $(\mathrm{p}<0,001)$. Observou-se, ainda, que as do GII iniciaram a gestação com quase $2,8 \mathrm{~kg}$ a mais $(\mathrm{p}=0,007)$ e que frequentaram mais consultas de $\mathrm{PN}(\mathrm{p}<0,001)$. Embora as adolescentes do GII tenham maior média de GPGT que as do GI, tal diferença não foi significativa $(p=0,317)$.

Estudos recentes ${ }^{25-27}$ apontam que entre as gestantes adolescentes há uma tendência a ganhar e a reter mais gordura do que entre as adultas e que esta é amplificada pela presença de franco crescimento materno, ou seja, em adolescentes jovens. Contudo, alegam que mesmo quando o crescimento materno já não é tão intenso há um contínuo acréscimo de gordura durante o terceiro trimestre de gestação. Consideram, portanto, que a adolescência e o terceiro trimestre gestacional sejam estágios que permitem a ocorrência da adipogênese.

Os resultados do presente estudo apontam que, em comparação às adolescentes que tiveram GPGT adequado, aquelas que encerraram a gestação com inadequação apresentavam maior média de peso pré-gestacional $(\mathrm{p}=0,006)$, maior média de IMC pré-gestacional $(\mathrm{p}=0,004)$ e maior média de ganho de peso no primeiro trimestre da gestação $(p=0,009)$ (Tabelas 3 e 4).

Confirmando tal achado, na análise da regressão de Poisson verificou-se que a intervenção não exerceu efeito positivo na adequação do GPGT ( $p>0,05$; Tabela 5), em modelo no qual foram controladas as variáveis: nível de instru- 
Tabela 1. Distribuição das puérperas adolescentes, segundo características sociodemográficas, estado nutricional pré-gestacional e adequação do ganho de peso gestacional total, nos grupos de estudo préintervenção (GI) e intervenção (GII). Rio de Janeiro, julho/04 a fevereiro/10.

\begin{tabular}{|c|c|c|c|c|c|}
\hline \multirow[b]{2}{*}{ Variáveis Maternas } & \multicolumn{2}{|c|}{ GI } & \multicolumn{2}{|c|}{ GII } & \multirow[b]{2}{*}{$\mathbf{p}$} \\
\hline & $\mathbf{n}$ & $\%$ & $\mathbf{n}$ & $\%$ & \\
\hline \multicolumn{6}{|l|}{ Nível de instrução } \\
\hline Ensino fundamental incompleto & 45 & 33,3 & 44 & 31,9 & $<0,001$ \\
\hline Ensino fundamental completo & 54 & 40,0 & 11 & 8,0 & \\
\hline Ensino médio incompleto & 19 & 14,1 & 60 & 43,5 & \\
\hline Ensino médio completo ou mais & 17 & 12,6 & 23 & 16,7 & \\
\hline \multicolumn{6}{|l|}{ Cor da pele } \\
\hline Branca & 45 & 46,4 & 36 & 26,1 & 0,001 \\
\hline Negra (preta ou parda) & 52 & 53,6 & 102 & 73,9 & \\
\hline \multicolumn{6}{|l|}{ Situação marital } \\
\hline Casada/vive com o companheiro & 9 & 9,6 & 50 & 39,7 & $<0,001$ \\
\hline Solteira/viúva & 85 & 90,4 & 76 & 60,3 & \\
\hline \multicolumn{6}{|c|}{ Condições de saneamento do domicílio } \\
\hline Adequada $^{\star a}$ & 62 & 93,9 & 135 & 94,4 & 0,893 \\
\hline Inadequada & 4 & 6,1 & 8 & 5,6 & \\
\hline \multicolumn{6}{|l|}{ Uso de cigarro na gestação } \\
\hline Sim & 14 & 12,3 & 6 & 4,2 & 0,016 \\
\hline Não & 100 & 87,7 & 138 & 95,8 & \\
\hline \multicolumn{6}{|l|}{ Uso de álcool na gestação } \\
\hline Sim & 13 & 11,5 & 11 & 7,6 & 0,290 \\
\hline Não & 100 & 88,5 & 133 & 92,4 & \\
\hline \multicolumn{6}{|c|}{ EN pré-gestacional $\left(\mathrm{IMC} \mathrm{kg} / \mathrm{m}^{2} / \text { idade) }\right)^{* b}$} \\
\hline Baixo peso & 2 & 1,4 & 3 & 5,3 & 0,152 \\
\hline Adequado & 121 & 87,1 & 45 & 78,9 & \\
\hline Sobrepeso & 8 & 5,8 & 2 & 3,5 & \\
\hline Obesidade & 8 & 5,8 & 7 & 12,3 & \\
\hline \multicolumn{6}{|l|}{ GPGT $(\mathrm{kg})^{\mathrm{c}}$} \\
\hline Adequado $^{d}$ & 47 & 29,4 & 44 & 30,6 & 0,822 \\
\hline Inadequado & 113 & 70,6 & 100 & 69,4 & \\
\hline
\end{tabular}

*a quando apresentava água tratada e encanada, rede de esgoto e coleta de lixo regular, sendo inadequada quando um dos serviços estava ausente. ${ }^{\star b} \mathrm{EN}=$ estado nutricional; $\mathrm{IMC}=$ índice de massa corporal $\left(\mathrm{kg} / \mathrm{m}^{2}\right)$. ${ }^{\star c} \mathrm{GPGT}=$ ganho de peso gestacional total. ${ }^{\star \mathrm{d}}$ quando dentro dos valores esperados segundo o estado nutricional pré-gestacional, conforme recomendação do Ministério da Saúde (2005).

ção, número de gestações, número de partos e número de consultas de PN. De acordo com os estudos de Nucci et al. ${ }^{28}$ e Padilha et al. ${ }^{8}$, realizados com mulheres brasileiras, as gestantes com desvio ponderal pré-gestacional apresentam maior risco de resultado obstétrico desfavorável, particularmente para a ocorrência de síndromes hipertensivas da gravidez, diabetes gestacional, micro e macrossomia fetal. Em estudo realizado com gestantes adolescentes americanas, a adequação do GPGT foi descrita em 47,8\%, enquanto que o ganho de peso excessivo foi descrito em $27,8 \%$ das adolescentes ${ }^{25}$.
Vale ressaltar que as recomendações de ganho de peso gestacional para adolescentes geralmente são construídas com base nas recomendações para adultas, tanto em relação aos pontos de corte de IMC pré-gestacional e gestacional $^{10,13,20}$, quanto às faixas de ganho de peso gestacional semanal e total. Analisando-se os valores de IMC propostos pela Organização Mundial da Saúde em $2007^{21}$, segundo a idade em anos e meses para meninas, verifica-se que são bem inferiores que os pontos de corte propostos para adultas. Tal interpretação pode ocasionar a classificação errônea da adolescente como, por exem- 
Tabela 2. Médias e desvios-padrão das variáveis maternas, obstétricas e da assistência pré-natal das gestantes adolescentes segundo grupos de estudo (GI e GII). Rio de Janeiro, julho/04 a fevereiro/10.

\begin{tabular}{|c|c|c|c|c|}
\hline Variáveis & $\mathbf{n}$ & Média $\pm \mathrm{DP}$ & Dif. Média & $\mathrm{p}^{\mathrm{a}}$ \\
\hline \multicolumn{5}{|l|}{ Idade materna no parto } \\
\hline GI & 160 & $17,5 \pm 1,40$ & $-0,272$ & 0,104 \\
\hline GII & 142 & $17,8 \pm 1,50$ & & \\
\hline \multicolumn{5}{|l|}{ Número de gestações } \\
\hline GI & 157 & $1,22 \pm 0,50$ & 0,077 & 0,122 \\
\hline GII & 144 & $1,14 \pm 0,37$ & & \\
\hline \multicolumn{5}{|l|}{ Número de partos } \\
\hline GI & 158 & $0,13 \pm 0,33$ & 0,078 & 0,016 \\
\hline GII & 144 & $0,05 \pm 0,21$ & & \\
\hline \multicolumn{5}{|l|}{ Número de abortos } \\
\hline GI & 67 & $0,24 \pm 0,50$ & 0,135 & 0,045 \\
\hline GII & 144 & $0,10 \pm 0,33$ & & \\
\hline \multicolumn{5}{|c|}{ Intervalo entre a última gestação e a atual (meses) } \\
\hline GI & 13 & $15,23 \pm 9,00$ & $-0,884$ & 0,613 \\
\hline GII & 13 & $18,85 \pm 11,70$ & & \\
\hline \multicolumn{5}{|c|}{ IG na primeira consulta $\mathrm{PN}$ (semanas) } \\
\hline GI & 152 & $14,50 \pm 4,79$ & $-2,164$ & $<0,001$ \\
\hline GII & 121 & $16,70 \pm 5,08$ & & \\
\hline \multicolumn{5}{|l|}{ Peso pré-gestacional (kg) } \\
\hline GI & 148 & $53,22 \pm 7,92$ & $-2,774$ & 0,007 \\
\hline GII & 140 & $56,00 \pm 9,45$ & & \\
\hline \multicolumn{5}{|l|}{ IMC pré-gestacional $\left(\mathrm{kg} / \mathrm{m}^{2}\right)$} \\
\hline GI & 150 & $21,23 \pm 2,90$ & $-0,620$ & 0,091 \\
\hline GII & 141 & $21,85 \pm 3,32$ & & \\
\hline \multicolumn{5}{|l|}{ Número de consultas PN } \\
\hline GI & 61 & $2,38 \pm 1,70$ & $-1,500$ & $<0,001$ \\
\hline GII & 144 & $3,90 \pm 1,64$ & & \\
\hline \multicolumn{5}{|c|}{ Ganho de peso gestacional total $(\mathrm{kg})$} \\
\hline GI & 160 & $13,66 \pm 5,90$ & $-0,642$ & 0,317 \\
\hline GII & 141 & $14,30 \pm 5,13$ & & \\
\hline
\end{tabular}

Legenda: $\mathrm{DP}$ = desvio-padrão; $\mathrm{ANPN}$ = assistência nutricional pré-natal; IG = idade gestacional; $\mathrm{PN}$ = pré-natal; IMC = índice de massa corporal $-\mathrm{kg} / \mathrm{m}^{2} .{ }^{a} \mathrm{~A}$ igualdade das variâncias foi observada por meio do teste de Levene $(\mathrm{p}>0,05)$.

Tabela 5. Resultados da análise multivariada por Regressão de Poisson para as razões de prevalência de inadequação do ganho de peso gestacional total, segundo os grupos de estudo $(\mathrm{GI}=542$ e GII = 204). Rio de Janeiro, julho/04 a fevereiro/10.

\begin{tabular}{lccccc}
\hline Variável & $\begin{array}{c}\text { Inadequação do ganho de } \\
\text { peso gestacional total \% }\end{array}$ & $\begin{array}{c}\mathbf{R P B}^{\mathbf{a}} \\
\left(\mathbf{I C}_{95 \%}\right)\end{array}$ & $\mathbf{p}$ & $\begin{array}{c}\mathbf{R P A}^{\mathbf{b}} \\
\left(\mathbf{I C}_{95 \%}\right)\end{array}$ & $\mathbf{p}$ \\
\hline $\begin{array}{l}\text { Grupo } \\
\text { GI }\end{array}$ & 70,6 & 1,017 & 0,823 & 0,957 & 0,591 \\
GII & 69,4 & $(0,878-1,179)$ & & $(0,816-1,123)$ & \\
\hline
\end{tabular}

${ }^{\mathrm{a}} \mathrm{RPB}=$ razão de prevalência bruta. ${ }^{\mathrm{b}} \mathrm{RPA}=$ razão de prevalência ajustada pelo modelo de regressão de Poisson controlada segundo as variáveis: nível de instrução, número de gestações, número de partos e número de consultas de pré-natal. 
Tabela 3. Características sociodemográficas, antropométricas e hábitos sociais maternos, segundo a adequação do ganho de peso gestacional total. Rio de Janeiro, julho/04 a fevereiro/10.

\begin{tabular}{|c|c|c|c|c|c|}
\hline \multirow[b]{3}{*}{ Variáveis } & \multicolumn{4}{|c|}{ Ganho de Peso Gestacional Total } & \multirow[b]{3}{*}{$\mathrm{p}$} \\
\hline & \multicolumn{2}{|c|}{ Adequado $^{a}$} & \multicolumn{2}{|c|}{ Inadequado } & \\
\hline & $\mathbf{n}$ & $\%$ & $\mathbf{n}$ & $\%$ & \\
\hline \multicolumn{6}{|l|}{ Condições de saneamento } \\
\hline Adequada & 59 & 29,9 & 138 & 70,1 & 0,716 \\
\hline Inadequada & 3 & 25,0 & 9 & 75,0 & \\
\hline \multicolumn{6}{|l|}{ Cor da pele } \\
\hline Branca & 22 & 27,2 & 59 & 72,8 & 0,523 \\
\hline Negra (parda e preta) & 48 & 31,2 & 106 & 68,8 & \\
\hline \multicolumn{6}{|l|}{ Situação marital } \\
\hline Casada/vive com companheiro & 42 & 31,1 & 93 & 68,9 & 0,919 \\
\hline Solteira & 27 & 31,8 & 58 & 68,2 & \\
\hline \multicolumn{6}{|l|}{ Nível de instrução } \\
\hline Não alfabetizada/EFI* & 24 & 27,0 & 65 & 73,0 & 0,677 \\
\hline Ensino fundamental completo & 22 & 33,8 & 43 & 66,2 & \\
\hline Ensino médio incompleto & 27 & 34,2 & 52 & 65,8 & \\
\hline Ensino médio completo e mais & 11 & 27,5 & 29 & 72,5 & \\
\hline \multicolumn{6}{|l|}{ Uso de cigarro } \\
\hline Sim & 8 & 40,0 & 12 & 60,0 & 0,343 \\
\hline Não & 71 & 29,8 & 167 & 70,2 & \\
\hline \multicolumn{6}{|l|}{ Uso de álcool } \\
\hline Sim & 5 & 20,8 & 19 & 79,2 & 0,269 \\
\hline Não & 74 & 31,8 & 159 & 68,2 & \\
\hline \multicolumn{6}{|l|}{ Uso de drogas ilícitas } \\
\hline Sim & 2 & 50,0 & 2 & 50,0 & 0,393 \\
\hline Não & 77 & 30,2 & 178 & 69,8 & \\
\hline \multicolumn{6}{|l|}{ IMC pré-gestacional $\left(\mathrm{kg} / \mathrm{m}^{2}\right)$} \\
\hline$<$ p5 (baixo peso) & 1 & 14,3 & 6 & 85,7 & 0,072 \\
\hline$\geq$ p5 $-<$ p 85 (eutrofia) & 77 & 32,3 & 161 & 67,7 & \\
\hline$\geq$ p85 - <p95 (sobrepeso) & 10 & 31,4 & 22 & 68,3 & \\
\hline$\geq$ p95 (obesidade) & 1 & 5,3 & 18 & 94,7 & \\
\hline
\end{tabular}

Legenda: $\mathrm{EFI}=$ ensino fundamental incompleto; $\mathrm{IMC}=$ índice de massa corporal $-\mathrm{kg} / \mathrm{m}^{2}$. ${ }^{\text {q }}$ quando mantido dentre os limites mínimo e máximo da faixa de ganho de peso recomendada segundo estado nutricional pré-gestacional.

plo, com IMC de baixo peso, o que subsidiará a orientação de ganho de peso acima do necessário, que para essa faixa de IMC é de 12,5 a $18 \mathrm{~kg}^{10,20}$, podendo favorecer a retenção do peso no pósparto e por conseguinte a obesidade no grupo.

A análise qualitativa dos dados permitiu a interpretação dos achados. Os sentidos emersos dos discursos das adolescentes entrevistadas revelaram importantes descompassos entre as orientações e as recomendações recebidas na intervenção e seus interesses e aspirações, motivo pelo qual experimentaram algumas condutas recomendadas, sem, contudo, adotarem o "plano alimentar" orientado, conforme será apresentado e discutido a seguir.
Facilidades e dificuldades para adesão às orientações nutricionais durante a gestação - a percepção das adolescentes

Chama a atenção o fato de que, pelos relatos das adolescentes entrevistadas, houve quase unanimidade em avaliar as orientações relacionadas à "comida" (alimentação e nutrição) como as mais difíceis de serem seguidas dentre todas as orientações recebidas no PN. Dessa forma, as adolescentes oferecem uma pista de que o conhecimento tecnocientífico pode tornar-se difícil de ser integrado às suas práticas alimentares, possivelmente por representar significados não compatíveis com seus costumes e valores sociocultu- 
Tabela 4. Médias e desvios-padrão das variáveis maternas, obstétricas e da assistência pré-natal das gestantes adolescentes, segundo a adequação do ganho de peso gestacional total. Rio de Janeiro, julho/04 a fevereiro/10.

\begin{tabular}{|c|c|c|c|c|}
\hline Variáveis & $\mathbf{n}$ & Média \pm DP & Dif. Média & $\mathbf{p}^{\mathrm{a}}$ \\
\hline \multicolumn{5}{|l|}{ Número de gestações } \\
\hline GPGT adequado & 91 & $1,24 \pm 0,54$ & 0,089 & 0,158 \\
\hline GPGT inadequado & 210 & $1,15 \pm 0,38$ & & \\
\hline \multicolumn{5}{|l|}{ Número de partos } \\
\hline GPGT adequado & 91 & $0,11 \pm 0,31$ & 0,029 & 0,414 \\
\hline GPGT inadequado & 211 & $0,08 \pm 0,27$ & & \\
\hline \multicolumn{5}{|l|}{ Número de abortos } \\
\hline GPGT adequado & 64 & $0,22 \pm 0,49$ & 0,103 & 0,128 \\
\hline GPGT inadequado & 147 & $0,12 \pm 0,34$ & & \\
\hline \multicolumn{5}{|c|}{ Intervalo entre a última gestação e a atual (meses) } \\
\hline GPGT adequado & 7 & $17,14 \pm 7,22$ & 0,143 & 0,976 \\
\hline GPGT inadequado & 19 & $17,00 \pm 11,50$ & & \\
\hline \multicolumn{5}{|c|}{ IG na primeira consulta $\mathrm{PN}$ (semanas) } \\
\hline GPGT adequado & 82 & $15,00 \pm 5,08$ & $-0,651$ & 0,327 \\
\hline GPGT inadequado & 191 & $15,65 \pm 5,00$ & & \\
\hline \multicolumn{5}{|l|}{ Peso pré-gestacional $(\mathrm{kg})$} \\
\hline GPGT adequado & 88 & $52,59 \pm 7,32$ & $-2,847$ & 0,006 \\
\hline GPGT inadequado & 200 & $55,44 \pm 9,28$ & & \\
\hline \multicolumn{5}{|l|}{ IMC pré-gestacional $\left(\mathrm{kg} / \mathrm{m}^{2}\right)$} \\
\hline GPGT adequado & 88 & $20,81 \pm 2,54$ & $-1,037$ & 0,004 \\
\hline GPGT inadequado & 203 & $21,84 \pm 3,30$ & & \\
\hline \multicolumn{5}{|c|}{ Ganho de peso no $1^{\circ}$ trimestre gestacional $(\mathrm{kg})$} \\
\hline GPGT adequado & 44 & $1,25 \pm 3,60$ & $-1,681$ & 0,009 \\
\hline GPGT inadequado & 97 & $2,93 \pm 3,47$ & & \\
\hline \multicolumn{5}{|l|}{ Peso ao nascer (gramas) } \\
\hline GPGT adequado & 90 & $3132,39 \pm 435,59$ & $-22,600$ & 0,709 \\
\hline GPGT inadequado & 207 & $3155,00 \pm 497,43$ & & \\
\hline \multicolumn{5}{|l|}{ IG no parto (DUM) } \\
\hline GPGT adequado & 80 & $38,81 \pm 1,89$ & 0,033 & 0,911 \\
\hline GPGT inadequado & 171 & $38,78 \pm 2,34$ & & \\
\hline \multicolumn{5}{|l|}{ Número de consultas PN } \\
\hline GPGT adequado & 88 & $7,05 \pm 1,93$ & $-0,486$ & 0,064 \\
\hline GPGT inadequado & 207 & $7,53 \pm 2,30$ & & \\
\hline
\end{tabular}

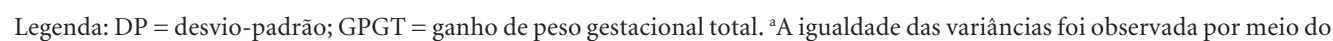
teste de Levene $(\mathrm{p}>0,05) .{ }^{1}$ quando mantido dentre os limites mínimo e máximo da faixa de ganho de peso recomendada segundo estado nutricional pré-gestacional

rais, influenciados, em geral, por familiares e membros de seu grupo social ${ }^{29,30}$.

Observaram, ainda, que "o que comiam" era uma questão com a qual "todo mundo ficava preocupado", fazendo referência a todos os profissionais de saúde que as atendiam. Apenas uma adolescente relatou não ter encontrado "dificuldade nenhuma” com as orientações do PN; no entanto, destaca-se que esta entrevistada somente compareceu à primeira consulta de nutrição e não mais retornou, alegando ter "fugido da nutrição" por considerar "chato" ter sua alimentação "regulada". A expressão "fugir da nutrição" emerge em seu discurso enquanto categoria de limite, como signo de preservação de sua intimidade na relação com o alimento. Assim, deixando de frequentar a consulta de nutrição, a adolescente mantém-se distante das normas e regras tecnocientíficas que oferecem restrição e controle à sua liberdade de escolha alimentar ${ }^{31}$. 
Ainda nas falas das entrevistadas, foi possível identificar motivações e obstáculos para a adesão cotidiana às recomendações dietéticas. Um dos principais motivos da não adesão foi "não gostar" ou "não ser chegada" a legumes e verduras, representados pelas categorias "coisas, coisinhas" nos discursos das adolescentes, que os reconheciam como alimentos dispensáveis e que lhes "provocavam vômito" quando se esforçavam a comê-los. Em oposição, a "comida mesmo" era representada pela composição de "arroz com feijão e bife", reconhecidos como alimentos "que tiravam a fome". Esses sentidos expressos pelas entrevistadas neste estudo são consistentes com os encontrados por outros pesquisadores, em estudos realizados com sujeitos de classes populares $^{32-34}$.

Como descrito no estudo de Baião e Deslan$\operatorname{des}^{34}$, as adolescentes que não trabalhavam ou estudavam durante a gestação dormiam e acordavam muito tarde, influenciando negativamente na adequação dos horários das refeições. No presente estudo, essa falta de "horário certo para comer", por sua vez, as induzia a "beliscar" alimentos com alta densidade energética ao longo do dia.

O controle do apetite foi outra dificuldade relatada por quase todas as entrevistadas, representando uma barreira ao consumo das quantidades de alimentos recomendadas pela nutricionista. Para as entrevistadas, ainda que sob a forma de aconselhamento, o plano alimentar tinha a principal conotação de um conjunto de regras que o nutricionista recomendava, mas que eram incompatíveis com as suas condições de grávidas e que, por isso, ficavam inconformadas.

Eu comia muito na gravidez e ela [a nutricionista] botou [no plano alimentar] meia concha de feijão e três colheres de arroz. Eu falei: ah, não dá. Assim vou passar fome. [...] eu era muito gulosa. Eu aumentei [o apetite] na gravidez. Me deu muita fome, muita fome, eu não podia ver nada que me dava vontade de comer [...] a tentação, é difícil. (E8)

$\mathrm{Na}$ visão de Anderson $^{35}$, para alguns grupos sociais a gravidez pode ser uma das poucas vezes na vida de uma mulher em que comer maiores quantidades de alimentos é aceito e até mesmo incentivado, mantendo-se o mito do "comer por dois".

No presente estudo, o excesso de ganho de peso somente passou a ser percebido pelas adolescentes como condição indesejada quando outros profissionais de saúde chamavam sua atenção para isso e, então, passava a se constituir em motivação para adesão às orientações do nutricionista.
[...] Assim..., porque eu nunca fui muito de comer muito legume, né? [risos]. [...]. Só no final [da gravidez] que eu comecei a me controlar um pouquinho [a alimentação] [risos] porque, é... a médica falou que..., eu acho que... em três semanas eu engordei três quilos, aí meu... caraca! Três quilos! Aí comecei a tentar seguir, né, direitinho, comi coisa até que eu não gosto: legume. Comia todos, é. (E3)

Todavia, não se pode negar que as entrevistadas se preocupavam com o ganho de peso excessivo, e nesse ponto se aproximavam das preocupações biomédicas, mas, isso não significa que o padrão de normalidade seja consensual. A normalidade identificada pela ferramenta matemática do cálculo do Índice de Massa Corpórea (IMC), do ganho de peso semanal e de sua adequação à idade gestacional, serve muito bem à lógica biomédica e não se ajusta facilmente ao mundo das adolescentes. Para estas, o valor desse ponto de corte entre a normalidade e o excesso de peso parece ser um pouco maior do que o considerado pela biomedicina. A faixa de sobrepeso classificada pela ciência tende a ser considerada pelas adolescentes como ganho de peso "naturalmente” esperado, por se tratar de algo próprio da gravidez. Neste sentido, a preocupação com o ganho de peso excessivo só se configura quando este se dá em um curto espaço de tempo.

Assim, apesar de os profissionais de saúde buscarem advertir a adolescente sobre os riscos que o ganho de peso gestacional excessivo pudesse trazer à sua saúde e à de seu bebê, sua percepção não se dava somente a partir dessa informação e, raramente, seria idêntica a que os profissionais tinham e tentavam fazer com que ela tivesse, pois esses riscos sempre seriam relativizados pelo permanente re(tecer) de sua significação.

Outro fator que contribuiu positivamente para a adesão das entrevistadas foi a participação do pai do bebê na consulta de nutrição e sua preocupação em dispensar cuidado e incentivo à adolescente, com atitudes de reforço da importância das orientações recebidas.

[...] meu marido ficava do lado, né, falando direto: Ah, porque você tem que se alimentar direito do jeito que a mulher tá falando, por causa do bebê. Aí eu comecei a seguir o que ele falava. E ele sempre falava, então, eu fui seguindo. (E2)

Estudiosos ${ }^{36,37}$ têm demonstrado que gestantes que não contam com a segurança e a companhia do parceiro durante a gestação, podem sofrer riscos físicos e psicológicos, especialmente quando são adolescentes. Nesse sentido, a influência positiva da presença do parceiro no desenvolvimento da gestação, no parto e no cuidado 
com a criança, é extremamente importante. Trazer à tona os valores dessa participação paterna significa a construção de significações e ressignificações da paternidade, especialmente quando esses pais também são adolescentes. Além disso, a inserção dos pais no contexto da saúde reprodutiva, com participação nas decisões e dividindo responsabilidades e cuidados com seu filho, significam o enfrentamento de preconceitos e estereótipos de gênero e a opção pela adoção de novos valores ${ }^{38}$.

\section{A consulta de nutrição na perspectiva das adolescentes}

Neste estudo, as adolescentes revelaram suas perspectivas sobre a consulta de nutrição, considerando o vínculo com as nutricionistas, a oportunidade de aprendizado, a possibilidade de promoção de mudanças em seus hábitos de vida e práticas alimentares, a duração e o nível de detalhamento da consulta e, por fim, o distanciamento entre o significado das ações produzidas e o sentido real e prático para os seus projetos existenciais.

Pode ser observado nos relatos das entrevistadas que as nutricionistas são percebidas como profissionais competentes, acolhedoras e interessadas em prestar uma atenção personalizada, favorecendo a formação de vínculo e a ampliação de seus conhecimentos.

Elas [as nutricionistas] são... muito boas. [...] Eu gostava [de ir à consulta de nutrição] porque... [...] eu ia pra médica e subia [para o ambulatório de nutrição, no andar superior ao de obstetrícia], eu gostava de vir. E gostava porque elas [as nutricionistas] me explicavam as coisas, elas explicavam as coisas pra mim. (E7)

Para algumas adolescentes, a consulta de nutrição, em especial, foi importante para o empreendimento de mudanças em rotinas diárias e práticas alimentares que as incomodavam.

Me sentia bem. Gostava de ir sempre [à consulta de nutrição]. Até pra me habituar aos horários [de acordar e das refeições], que eu não tinha. [...] hoje em dia, eu tenho sempre o horário certo de tomar café, almoçar, tudo direitinho. Porque, antes, eu não fazia nada disso. (E2)

Entretanto, o longo tempo da consulta foi um aspecto avaliado negativamente, pois sua duração podia ultrapassar sessenta minutos. Também foi motivo de insatisfação a quantidade excessiva de perguntas e o alto grau de detalhamento sobre o quê, como, quanto, onde e quando se alimentavam, deixando-as "cansadas" e "com fome". O "cansaço" sintetizava o descon- solo e a impaciência das adolescentes que se reconheciam como sujeitos de uma ação que buscava desnudar e avaliar suas escolhas, para, em seu desfecho, apresentar considerações racionais que conformariam um discurso técnico permeado por regras e coerções. O termo "fome" indicava que a consulta se estendia até bem próximo ao horário do almoço e que, inexoravelmente, focalizava alimentos e preparações, aguçando ainda mais essa sensação.

O estudo de Líbera et al. ${ }^{39}$, que avaliou a assistência PN na mesma maternidade, mas sob a perspectiva de puérperas adultas, mostrou que a consulta de nutrição para essas mulheres a satisfaziam pelo diálogo, acolhimento e incentivo que recebiam do nutricionista, sem que o tempo e o volume de questionamentos necessários para isso as incomodassem tanto quanto incomodou às adolescentes.

Sob o ponto de vista de algumas adolescentes, o incômodo vivenciado em uma consulta longa, que primava pelo detalhamento extremo de suas práticas alimentares, assumia o mesmo significado do "enjôo" típico da gravidez, tão indesejado pelo grande desconforto que é capaz de causar.

Eu achei demorado. Eu achei um pouco enjoado, que é muita pergunta e nem sempre eu lembrava quantas vezes eu comia no mês [cada tipo de alimento], quantas colheres eu comia. É bem demorado, mas, é bem útil. É um chato, mas que é necessário entendeu? (E10)

Esse significado construído pela adolescente reflete sua apreensão biologizada e em completa sintonia com o contexto biológico e pragmático da consulta. Como se vê, o "academicismo" inscrito nas práticas em nutrição geralmente acarreta em uma aplicação sistemática da dimensão biometabólica, com a adoção de modelos e instrumentos engessados, padronizados e cansativos. Isso significa dizer que, apesar dos avanços na busca da horizontalização da relação e na criação de vínculo com as gestantes, o nutricionista perde-se na incompreensão da multidimensionalidade dos condicionantes das práticas alimentares e termina por render-se a uma abordagem unidimensional e exclusivamente biológica, com conteúdo tecnicamente envernizado ${ }^{40}$.

Promover e orientar práticas alimentares saudáveis e adequadas para o ganho de peso gestacional considerando as variações individuais quanto às necessidades em cada gestação, são aspectos fundamentais da prática do nutricionista para a prevenção de agravos à saúde materna e fetal ${ }^{4-6}$. Porém, cabe ressaltar que os mé- 
todos utilizados para avaliação do consumo alimentar, especialmente a frequência semiquantitativa, pode tornar a consulta de nutrição muito prolongada, repetitiva e previsível. As adolescentes são convidadas a responder repetidas vezes a indagações que obedecem a um roteiro rígido, cuja linearidade imposta ocupa o espaço que teriam para expressarem-se livremente. A burocracia tecnocientífica, soberana aos seus sentimentos, interesses e desejos, intensifica a angústia, a chateação e a impaciência das adolescentes, negando-lhes a possibilidade de participarem conscientemente do processo de tomada de decisão sobre o seu cuidado nutricional.

[...] quase toda consulta era a mesma coisa. [A nutricionista] Só me pesava, me perguntava o que eu comia um dia antes... só isso. Agora, no grupo [de atendimento multidisciplinar] eu gostava mais do que... a consulta particular [se referindo à consulta individual com a nutricionista]. [...] Gostava mais porque era todo mundo, a gente conversava sobre a gente. (E11)

Nas falas das entrevistadas observa-se a ideia de monotonia da consulta de nutrição associada à centralidade da mensuração e avaliação do consumo alimentar e pela ênfase conferida ao controle do ganho de peso. Dessa forma, a consulta tende a se caracterizar pelo aborrecimento, pelo tédio, em oposição ao atendimento em grupo, cuja centralidade é da adolescente, da sua narrativa e das de outras gestantes adolescentes.

Diante do exposto, acredita-se que há a necessidade da reinvenção da prática clínica do nutricionista e de transformá-la em "trabalho em saúde”, essencialmente realizado em um espaço de intercessão partilhada ${ }^{41}$ com as gestantes, oferecendo um cuidado nutricional que vincule a promoção de práticas alimentares saudáveis ao prazer e ao bem-estar, ocupando o lugar de parceiro na construção de seus projetos de felicidade ${ }^{42}$.

Esse "cuidado", como defende Ayres ${ }^{41}$, se estrutura como potencial reconciliador entre as práticas assistenciais e a vida por meio da (re)articulação entre a técnica e os elementos emersos da experiência pessoal e social dos sujeitos em interação - gestantes adolescentes e nutricionista. Dessa forma, consolida-se em um cuidado pleno, que contempla o êxito técnico - o cuidado nutricional contribuindo na melhoria das condições de saúde das gestantes e seus filhos - e o eleva à condição de sucesso prático - as orientações nutricionais ganham um sentido mais aproximado dos valores, vivências e cotidiano das gestantes, que as apreendem como conquista, como fonte de realização, de prazer e de felicidade ${ }^{43}$.
Este estudo apresenta duas limitações, uma relacionada à utilização, em sua primeira fase, de dados coletados em prontuários, nos quais a ausência de informações de puérperas que não haviam realizado o PN na maternidade estudada acarretaram em um número considerável de missing values. A outra limitação se refere à perda de informações, dentre as quais o ganho de peso gestacional total, de algumas gestantes acompanhadas na coorte, mas que tiveram seus partos em outra maternidade.

Todavia, apesar da impossibilidade de extrapolação dos êxitos e das dificuldades, o presente artigo oferece informações sobre situações cotidianas da atenção nutricional a gestantes, sobretudo adolescentes. Isso é relevante pela importante influência que o estado nutricional materno exerce sobre o prognóstico da gravidez, especialmente na adolescência ${ }^{2,4,6}$.

\section{Considerações finais}

Em busca da construção de uma síntese ampliada, ou seja, do diálogo conciso e coerente com a plurideterminação do objeto investigado, os dados do presente estudo apontam, dentre outros aspectos, que um dos êxitos técnicos (metas) da atenção nutricional da gestante adolescente, qual seja, o de possibilitar a adequação do ganho de peso gestacional, ainda é um desafio. Cabe destacar que o principal achado da dimensão quantitativa deste estudo foi a não verificação de impacto significativamente positivo da intervenção sobre a adequação do GPGT das adolescentes acompanhadas pela nutricionista ao longo da gestação, quando comparadas às adolescentes do grupo controle histórico. Tal resultado, no entanto, passa a ser considerado "previsível", quando se abre o espaço para ouvir a "voz" daquelas para as quais as ações estão voltadas e das quais depende o sucesso técnico dessas ações - a gestante adolescente. As percepções que emergiram das falas das adolescentes oferecem valiosas pistas do quão limitadas são as práticas essencialmente técnicas e interessadas apenas no tratar, no prescrever e no controlar. A dominância da abordagem prescritiva-normativa, ainda presente no aconselhamento nutricional, representa um obstáculo para que a adolescente, mesmo que gestante, ponha em prática as orientações a ela oferecidas.

A complementação dos resultados quantitativos pelas nuances qualitativas produziu uma interface entre esses dois planos de análise que possibilitou avaliar não só o impacto da atenção 
nutricional, mas, sobretudo, desvelar as subjetividades que modulavam o grau de envolvimento e participação das adolescentes nessa intervenção.

A experiência relatada neste artigo destaca a importância e a necessidade de que a atenção nutricional a gestantes adolescentes seja oferecida no início do PN e que se estenda ao longo da gestação. Mas, sinaliza, também, para a necessidade de que, sem descuidar do conhecimento e da competência técnica, haja a incorporação de uma sabedoria prática de compartilhamento e de dialogicidade, para permitir à adolescente agir e refletir sobre a orientação que será coproduzida. Assim se alcançará o cuidado.

Com a trajetória analítica e reflexiva aqui desenvolvida, acredita-se ter contribuído para, além e junto do necessário, avançar na perspectiva da reavaliação do instrumental tecnocientífico balizador da assistência nutricional, avançar, também, na perspectiva de transformá-la em uma práxis capaz de gerar processos orientados para a tomada de decisões compartilhadas.

\section{Colaboradores}

MMAS Santos participou de todas as etapas de elaboração do artigo. C Saunders e DC Barros participaram da análise dos dados quantitativos e da discussão dos resultados. MR Baião participou da análise e interpretação dos dados qualitativos. Todos os autores participaram da elaboração do artigo final. 


\section{Referências}

1. Guerra AFFS, Heyde MED, Mulinari RA. Impacto do estado nutricional no peso ao nascer de recém nascidos de gestantes adolescentes. Rev Bras Ginecol Obstet 2007; 29(3):126-133.

2. Furlan JP, Guazzelli CAF, Papa ACS, Quintino MP, Soares RVP, Mattar R. A influência do estado nutricional da adolescente grávida sobre o tipo de parto e o peso do recém-nascido. Rev Bras Ginecol Obstet 2003; 25(9):625-630.

3. Santos GHN, Martins MG, Sousa MS. Gravidez na adolescência e fatores associados com baixo peso ao nascer. Rev Bras Ginecol Obstet 2008; 30(5):224-231.

4. Saunders C, Accioly E, Costa RSS, Lacerda EMA, Santos MMAS. Gestante Adolescente. In: Accioly E, Saunders C, Lacerda EMA, organizadores. $\mathrm{Nu}$ trição em Obstetrícia e Pediatria. Rio de Janeiro: Cultura Médica; 2009. p. 149-172.

5. Belarmino GO, Moura ERF, Oliveira NC, Freitas GL. Risco nutricional entre gestantes adolescentes. Acta paul. enferm 2009; 22(2):169-175.

6. Pencharz PB. Special problems of nutrition in the pregnancy of teenagers. In: Hornstra G, Uauy R, Yang X, editors. The impact of maternal nutrition on the offspring. Nestlé Workshop Series Pediatric Program 2005; 55:213-220.

7. Scholl TO, Miller LK, Salmon RW, Cofsky MC, Shearer J. Prenatal care adequacy and the outcome of adolescent pregnancy: effects on weith gain, preterm delivery, and birth weigth. Obstet Gynecol 1987; 69(3 Pt 1):312-316.

8. Padilha PC, Saunders C, Machado RCM, Silva CL, Bull A, Sally EOF, Accioly E. Associação entre o estado nutricional pré-gestacional e a predição do risco de intercorrências gestacionais. Rev Bras Ginecol Obstet 2007; 29(10):511-518.

9. Brasil. Ministério da Saúde (MS). Secretaria de Atenção à Saúde. Departamento de Ações Programáticas Estratégicas. Área Técnica de Saúde da Mulher. Pré-Natal e Puerpério: atenção qualificada e humanizada - manual técnico. 3a Edição Revisada. Brasília: MS; 2006.

10. Institute of Medicine; National Research Council. Weight Gain During Pregnancy: Reexamining the Guidelines. Washington (DC): National Academy of Science; 2009.

11. Institute of Medicine; National Research Council. Influence of pregnancy weight on maternal and child health: Workshop report. Washington: National Academy Press; 2007.

12. Olson CM, Strawderman MS. Modifiable behavioral factors in a biopsychosocial model predict inadequate and excessive gestational weight gain. J Am Diet Assoc 2003; 103(1):48-54.

13. Insitute of Medicine (IOM). Nutrition during pregnancy. Washington: National Academy Press; 1990.

14. Polley BA, Wing RR, Sims CJ. Randomized controlled trial to prevent excessive weight gain in pregnant women. Int J Obes Relat Metab Disord 2002; 26(11):1494-1502.

15. Rouse DJ. Potential Cost-Effectiveness of Nutrition Interventions to Prevent Adverse Pregnancy Outcomes in the Developing World. J Nutr 2003; 133(Supl. 2):1640S-1644S.
16. Deslandes SF, Assis SG. Abordagens quantitativa e qualitativa em saúde: um diálogo das diferenças. In: Minayo MCS, Deslandes SF, organizadores. $\mathrm{Ca}$ minhos do pensamento - Epistemologia e método. Rio de Janeiro: Fiocruz; 2002. p. 195-226.

17. Silverman D. O que é pesquisa qualitativa? In: Interpretação de dados qualitativos: métodos para análise de entrevistas, textos e interações. Porto Alegre: Artmed; 2009. p. 42-66.

18. Rodrigues EM, Soares FPTP, Boog MCF. Resgate do conceito de aconselhamento no contexto do atendimento nutricional. Rev. Nutr. 2005; 18(1):119-128.

19. Libera BD, Baião MR, Santos MMAS, Padilha PC, Alves PD, Saunders C. Adherence of pregnant women to dietary counseling and adequacy of total gestational weight gain. Nutr Hosp 2011; 26(1):96102.

20. Brasil. Ministério da Saúde (MS). Secretaria de Atenção à Saúde. Departamento de Ações Programáticas Estratégicas. Área Técnica de Saúde da Mulher. Pré-Natal e Puerpério: atenção qualificada e humanizada - manual técnico. Brasília: MS; 2006.

21. World Health Organization (WHO). WHO reference 2007: Growth reference data for 5-19 years. [acessado 2008 set 18]. Disponível em: http://www.who. int/growthref/en/ 2007.

22. Brasil. Ministério da Saúde (MS). SISVAN. Protocolos do Sistema de Vigilância Alimentar e Nutricional - SISVAN na assistência à saúde. Brasília: MS; 2008.

23. Schwandt TA. Três posturas epistemológicas para a investigação qualitativa. In: Denzin NK, Lincoln YS, organizadores. O planejamento da pesquisa qualitativa: teorias e abordagens. Porto Alegre: Artmed; 2006. p. 193-217.

24. Bardin L. Análise de conteúdo. Lisboa: Edições 70; 2008.

25. Oken E, Rifas-Shiman SL, Field AE, Frazier AL, Gillman MW. Maternal Gestational Weight Gain and Offspring Weight in Adolescence. Obstet Gynecol 2008; 112(5):999-1006.

26. Jones R, Cederberg H, Wheeler S, Poston L, Hutchinson C, Seed P, Oliver RL, Baker PN. Relationship between maternal growth, infant birthweight and nutrient partitioning in teenage pregnancies. BJOG 2010; 117(2):200-211.

27. Scholl TO. Adolescent pregnancy: an overview in developed and developing nations. Perinatol Reprod Hum 2007; 21:193-200.

28. Nucci LB, Schmidt MI, Duncan BB, Fuchs SC, Fleck ET, Britto MMS. Nutritional status of pregnant women: prevalence and associated pregnancy outcomes Rev Saude Publica 2001; 35(6):502-507.

29. Mintz SW. Comida e antropologia: uma breve revisão. Rev. bras. Ci. Soc. 2001; 16(47):31-42.

30. Canesqui AM, Garcia RWD. Uma introdução à reflexão sobre a abordagem sociocultural da alimentação. In: Canesqui AM, Garcia RWD, organizadores. Antropologia e nutrição: um diálogo possível. Rio de Janeiro: Fiocruz; 2005. p. 9-19.

31. Poulain J-P, Corbeau J-P. Libres mangeurs? In: Courbeau J-P, Poulain J-P, organizadores. Penser l'alimentation: entre imaginaire et rationalité. Toulouse: Éditions Privat; 2002. 
32. Canesqui AM. A qualidade dos alimentos: análise de algumas categorias da dietética popular. Rev. Nutr. 2007; 20(2):203-216.

33. Rotenberg S, De Vargas S. Práticas alimentares e o cuidado da saúde: da alimentação da criança à alimentação da família. Rev. Bras. Saude Mater. Infant. 2004; 4(1):85-94.

34. Baião MR, Deslandes SF. Práticas alimentares na gravidez: um estudo com gestantes e puérperas de um complexo de favelas do Rio de Janeiro (RJ, Brasil). Cien Saude Colet 2010; 15(Supl. 2):3199-3206.

35. Anderson AS. Pregnancy as a time for dietary change? Proc Nutr Soc 2001; 60(4):497-504.

36. Costa COM, Lima IC, Martins Júnior DF, Santos AST, Araújo FPO, Assis DR. Gravidez na adolescência e co-responsabilidade paterna: trajetória sociodemográfica e atitudes com a gestação e a criança. Cien Saude Colet 2005; 10(3):719-727.

37. Lyra J. Participação masculina na gravidez adolescente. In: Vieira EM, Fernandes MEL, Bailey P, Mckay A, organizadores. Seminário gravidez na adolescência. Rio de Janeiro: Associação Saúde da Família; 1998. p. 119-126.

38. Barreto ACM, Almeida IS, Ribeiro IB, Tavares KFA. Paternidade na Adolescência: tendências da produção científica. Adolesc Saude 2010; 7(2):54-59

39. Líbera BD, Saunders C, Santos MMAS, Rimes KA, Brito FRSS, Baião MR. Avaliação da assistência prénatal na perspectiva de puérperas e profissionais de saúde. Cien Saude Colet 2010: 16(12):4855-4864.

40. Garcia RWD. Representações sociais da alimentação e saúde e suas repercussões no comportamento alimentar. Physis 1997; 7(2):51-68.

41. Ayres JRCM. Cuidado e reconstrução das práticas de saúde. Interface Comun Saúde Educ 2004; 8(14): 73-91.

42. Merhy EE. A perda da dimensão cuidadora na produção da saúde: uma discussão do modelo assistencial e da intervenção no seu modo de trabalhar a assistência. In: Campos CR, Malta D, Reis A, organizadores. Sistema Único de Saúde em Belo Horizonte: reescrevendo o público. Belo Horizonte: Xamã; 1998. p. 103-120.

43. Ayres JRCM. Cuidado e humanização das práticas de saúde. In: Deslandes SF, organizador. Humanização dos cuidados em saúde: conceitos, dilemas e práticas. Rio de Janeiro: Editora Fiocruz; 2006. p. 49-83.

Artigo apresentado em 24/10/2011

Aprovado em 09/01/2012

Versão final apresentada em 08/02/2012 